\title{
Early pubertal onset and its relationship with sexual risk taking, substance use and anti-social behaviour: a preliminary cross-sectional study Jennifer Downing* and Mark A Bellis
}

\author{
Address: Centre for Public Health, Liverpool John Moores University, Liverpool, UK \\ Email: Jennifer Downing* - j.downing1@ljmu.ac.uk; Mark A Bellis - m.a.bellis@ljmu.ac.uk \\ * Corresponding author
}

Published: 3 December 2009

BMC Public Health 2009, 9:446 doi:I0.I 186/147/-2458-9-446

This article is available from: http://www.biomedcentral.com/I47I-2458/9/446

(c) 2009 Downing and Bellis; licensee BioMed Central Ltd.

This is an Open Access article distributed under the terms of the Creative Commons Attribution License (http://creativecommons.org/licenses/by/2.0), which permits unrestricted use, distribution, and reproduction in any medium, provided the original work is properly cited.
Received: 7 June 2009

Accepted: 3 December 2009

\begin{abstract}
Background: In many countries age at pubertal onset has declined substantially. Relatively little attention has been paid to how this decline may affect adolescent behaviours such as substance use, violence and unprotected sex and consequently impact on public health.

Methods: In the UK, two opportunistic samples (aged 16-45 years), paper-based $(\mathrm{n}=976)$ and online $(n=1117)$, examined factors associated with earlier pubertal onset and whether earlier age of onset predicted sexual risk-taking, substance use and anti-social behaviours during early adolescence.
\end{abstract}

Results: Overall, $45.6 \%$ of females reported menarche $\leq 12$ years and $53.3 \%$ of males were categorised as having pubertal onset $\leq \mathrm{II}$ years. For both sexes earlier pubertal onset was associated with poorer parental socio-economic status. Other pre-pubertal predictors of early onset were being overweight, more childhood illnesses (females) and younger age at time of survey (males). For both sexes earlier puberty predicted having drunk alcohol, been drunk, smoked and used drugs $<14$ years as well as having a sexual debut and unprotected sex $<16$ years. Males with earlier pubertal onset were more likely to report fighting and aggressive responses to emotional upset during early adolescence while females were more likely to report being bullied and having taken more time off school.

Conclusion: Results provide sufficient evidence for changes in age of pubertal onset to be further explored as a potential influence on trends in adolescent risk behaviours. Further insight into the relationship between early puberty and both obesity and socio-economic status may help inform early interventions to tackle the development of risk behaviours and health inequalities during early adolescence.

\section{Background}

Age of pubertal onset has decreased over recent decades $[1,2]$ with boys in many developed countries beginning puberty aged between 9.5 and 12 years and girls around 13 years [3]. While menarche, a distinctive marker of puberty in girls, now occurs typically around 12 to 13 years in developed countries [4,5], 150 years ago average age was substantially higher (i.e. over 15 years) [6]. A variety of reasons for this decrease have been suggested, including changes in family structure (e.g. father absence) 
[7], reductions in levels of childhood illness, better nutrition and increased obesity in childhood [8].

Of concern for public health is that while current age of physical maturation has declined, this has not been matched by developments in the social support and education children may require at increasingly earlier ages [8]. Consequently, psychosocial maturity (i.e. social puberty) may now lag behind physical development by a greater period than at any time previously in human history $[1,8]$. Such a temporal dissociation is not without health consequences. Earlier pubertal onset has been associated with increased anxiety as well as concerns with body shape, weight [9] and the development of eating disorders [10]. Moreover, in the absence of psychosocial maturity, exploratory behaviours that mark early adolescence may result in uninformed risk-taking behaviours. Early maturers are more likely to adopt deviant behaviours including, for example, increased use and dependence on alcohol [11]. In girls, such effects may result from association with deviant peers, older individuals and boyfriends [12]. Mature appearance in girls at age 13 has also been related to a greater number of sexual partners by age 16 years [13]. A high rate of partner change is associated with subsequent increased risks of sexually transmitted infections [14] and early sexual initiation is associated with teenage pregnancy [15]. For boys, relationships between early maturation and association with deviant peers are unclear $[12,16]$. However, level of physical maturity is positively associated with engagement in violence, property crime, illicit drug use and early sexual behaviour [16].

Across a wide range of countries levels of sexually transmitted infections in teenagers continue to escalate $[17,18]$. In addition, levels of alcohol misuse, even during early teenage years, are high in many countries across Europe with the proportions reporting drunkenness in the last 30 days ranging from $31 \%$ in Iceland to $80 \%$ in Austria (aged 15-16 years) [19]. Furthermore, despite a recent reduction in reported levels of cannabis use, 23\% and $17 \%$ of European boys and girls respectively report having ever used illicit drugs [19]. While limited studies have explored relationships between pubertal timing and sex $[13,20,21]$, substance use $[11,12,22]$, and other risk behaviours [10,23], the potential impact of earlier puberty on public health remains relatively unexamined. Further, no studies have investigated how adolescent risktaking relates to early puberty in the UK. However, the UK has one of the highest proportions of sexually active 15 year olds across all high income countries with $40 \%$ reporting sexual activity compared to $15-28 \%$ across other OECD (Organisation for Economic Cooperation and Development) nations [24]. Moreover, the UK also experiences one of the highest teenage pregnancy rates in Europe [25]. Over 30\% of young people in the UK report ever being drunk two or more times compared to an average of $15 \%$ across other OECD nations (samples include ages 11,13 and 15 years) [24], and for those aged 15-16 the UK reports some of the highest levels of illicit drug use in Europe [19].

While sexual risk-taking, substance using and related antisocial behaviours emerge, in general, as individuals pass through puberty [26], such behaviours are present at disproportionate rates in more deprived populations [2732]. However, relationships between pubertal timing and inequalities (especially in health) have received relatively little attention. Consequently, here we retrospectively measure relationships between early onset puberty in UK males and females in order to examine whether earlier puberty is associated with greater participation in risk-taking behaviour during adolescence. Furthermore, we explore individual and family characteristics associated with age of pubertal onset to identify factors (e.g. obesity) that may contribute to earlier puberty and therefore increase the gap between physical and social development.

\section{Methods}

Previously established factors influencing age of pubertal onset were identified through literature searches and questions developed to measure which of these factors relate to earlier puberty in our sample. Thus, respondents provided details of current sexuality [33] and ethnicity [34] and for the period prior to menarche (for females) and prior to age 12 years (for males: an average used for pubertal onset in the absence of a distinct physiological marker) questions measured weight (self-assessed as under, over or about average weight and dichotomised into overweight and not overweight) [5], family structure (i.e. which parents or other guardians they were living with) $[7,35]$, time spent off school and history of major childhood illness. For the latter variable typical childhood illness were listed (i.e. measles, mumps, chicken pox, whooping cough, pneumonia) but individuals could also report any other illness they suffered during childhood and regarded as major (e.g. meningitis). For socio-economic status, we collected occupation of respondents' parents or main carer also before they reached menarche/ 12 years of age and coded these to Office for National Statistics socio-economic classification codes $[36,37]$. To examine the effects of deprivation and avoid small sub groups, these categories were dichotomised into deprived (code 5; semi-routine and routine occupations, never worked and long-term unemployed) and not deprived (codes 1-4: managerial and professional occupations; intermediate occupations; small employers and own account workers and; lower supervisory and technical occupations respectively). For each respondent, final socio-economic status at pubertal onset was based on the 
highest ranked parent or carer with whom respondents lived.

Age at onset of risk behaviour was assessed by examining age of first (if ever); alcohol use, being drunk, drug use, smoking and having sex. Questions examining sex also addressed; whether first sex was under the influence of drugs or alcohol; age that individuals had sex without any contraception; and, for girls, age of first pregnancy and abortion (if any). For measures of aggression, questions addressed typical responses to periods of unhappiness, frequency of fighting and, bullying and being bullied at school (i.e. after menarche, females; $>12$ years, males). Previously validated questions were incorporated wherever possible.

Although puberty begins before menarche, age at menarche is a useful biological proxy for age of pubertal onset [38] and consequently we utilise age of menarche as our measure for pubertal onset in girls. For males, although prospective studies often utilise pubic and facial hair growth, voice change, and genital growth these can be unreliable [38] and may be poorly recalled in a retrospective study. Previous retrospective studies have disregarded such measures and examined self-perception of pubertal onset [22]. For males we recorded age of first sexual attraction, first nocturnal emission (i.e. wet dream) and first masturbation [15]. Males were allocated a proxy pubertal onset age as the youngest age taken from all questions that they answered. Our measures for males are likely to produce a peri-pubertal age that is not directly comparable to age at menarche in females $[8,39]$. Therefore throughout this paper, analyses are separated by sex.

Both male and female questionnaires were piloted on individuals within the target age range (16-45 years). As an initial exploration of relationships between early pubertal onset and adolescent risk behaviours, two different populations (one paper based and one online) were surveyed. Ethical approval was given by Liverpool John Moores University (LJMU) research ethics committee for their distribution in both paper-based and online formats with a target sample size of 1000 in each format. Opportunistic rather than representative samples were sought as analyses aimed to explore interaction between variables relating to an individual rather than measure, or extrapolate to, population levels. The paper-based questionnaires were disseminated to students and staff at LJMU and the University of Liverpool (sampling, 15th January to $24^{\text {th }}$ September 2008). The study was explained to all potential participants and an information sheet, consent form and a copy of the questionnaire provided to each prior to them agreeing to participate. All questionnaires were anonymous and, on completion, were either handed to researchers in a sealed envelope or, were taken away by participants and posted back in a pre-paid envelope. The online questionnaire utilised identical questions and allowed collection of an independent comparative sample (sampling, 8th August to $4^{\text {th }}$ December 2008). This was advertised on websites (e.g. Men's Health forum; Youth Information) and distributed by email via members of organisations (e.g. Sex Education forum) as well as through newsletters.

A total of 1923 paper questionnaires were distributed with 1097 questionnaires completed and returned ( $57.0 \%$ response). Any access to the questionnaire online was monitored regardless of whether individuals then completed the questions. In total, access to the online questionnaire occurred 2183 times and resulted in 1242 completions (56.9\% response). Overall, 236 questionnaires were excluded as they had been completed by nonUK residents. A further eight were excluded as key fields, such as age or sex, were missing or age fell outside the target range. Two cases were excluded on the basis of spoilt returns resulting in final samples for analysis of 976 paper based (female 580; male 396) and 1117 online (female 823, male 294) respondents. Sample sizes vary slightly between analyses where not all questions have been completed by all respondents. Sample sizes are included with each analysis (see tables 1, 2, 3, 4 \&5) in order to clarify response rates and numbers being analysed. Data were analysed using SPSS v14 and EpiStat. Analyses used chi square and employed backward conditional logistic regression and multinomial logistic regression to adjust for confounding factors. For both females and males paper and online samples differed significantly by age, sexuality, levels of childhood illness and socio-economic status (table 1). Consequently, in bivariate analyses online and paper samples have been treated separately and in multivariate analyses collection method has been included as an independent variable.

\section{Results}

Females were categorised into either earlier $(\leq 12$ years; $45.6 \%$ ) or later ( $\geq 13$ years; $54.4 \%$ ) puberty, and males into pubertal onset $\leq 11$ years $(53.3 \%)$ or $\geq 12$ years (46.7\%). Tables 2 (female) and 3 (male) explore which demographics, health and other factors (relating to life before menarche $/<12$ years) are associated with respondents' pubertal timing. For females, both online and paper based respondents showed a significant association between being overweight and earlier puberty (table 2). Levels of childhood illness and poorer parental socio-economic status did not reach significance for paper-based or online surveys separately. However, these relationships were significant using logistic regression (LR) analysis on the combined data sets (table 2); with those from poorer socio-economic groups or those who had experienced more childhood illnesses being more likely to experience 
Table I: Demographic and pre-pubertal indicators by sample type and sex

\begin{tabular}{|c|c|c|c|c|c|c|c|c|c|}
\hline & & \multicolumn{4}{|c|}{ Female } & \multicolumn{4}{|c|}{ Male } \\
\hline & & $\mathbf{n}$ & Online & Paper & $\mathbf{P}$ & $\mathbf{n}$ & Online & Paper & $\mathbf{P}$ \\
\hline Age at & 16-24 years & 840 & 42.0 & 85.6 & $* * *$ & 506 & 48.6 & 91.9 & $* * *$ \\
\hline Survey & $\geq 25$ years & 560 & 58.0 & 14.4 & & 183 & 51.4 & 8.1 & \\
\hline \multirow[t]{2}{*}{ Ethnicity } & white & 1339 & 96.6 & 94.8 & 0.100 & 650 & 93.5 & 95.4 & 0.271 \\
\hline & other & 58 & 3.4 & 5.2 & & 37 & 6.5 & 4.6 & \\
\hline \multirow[t]{2}{*}{ Sexuality } & heterosexual & 1276 & 91.7 & 95.5 & $* *$ & 570 & 70.6 & 92.1 & $* * *$ \\
\hline & Lesbian/bisexual & 93 & 8.3 & 4.5 & & 117 & 29.4 & 7.9 & \\
\hline Number of childhood & $\leq \mathrm{I}$ & 949 & 61.6 & 76.6 & $* * *$ & 505 & 64.6 & 79.7 & $* * *$ \\
\hline illnesses $\S$ & $\geq 2$ & 451 & 38.4 & 23.4 & & 184 & 35.4 & 20.3 & \\
\hline \multirow[t]{2}{*}{ Overweight§ } & no & 1197 & 85.7 & 85.4 & 0.872 & 617 & 91.8 & 88.3 & 0.129 \\
\hline & yes & 201 & 14.3 & 14.6 & & 70 & 8.2 & II.7 & \\
\hline Father at & no & 297 & 20.0 & 21.7 & 0.455 & 143 & 19.1 & 23.6 & 0.162 \\
\hline home $\S$ & yes & 1070 & 80.0 & 78.3 & & 517 & 80.9 & 76.4 & \\
\hline Days off & $\leq 10$ & 1122 & 82.4 & 80.1 & 0.284 & 546 & 81.4 & 80.3 & 0.699 \\
\hline school per year§ & $\geq \mathrm{II}$ & 256 & 17.6 & 19.9 & & 130 & 18.6 & 19.7 & \\
\hline In poorest socio- & no & 916 & 64.0 & 78.0 & $* * *$ & 482 & 68.7 & 78.3 & ** \\
\hline economic grouping $\$ \S$ & yes & 399 & 36.0 & 22.0 & & 167 & 31.3 & 21.7 & \\
\hline
\end{tabular}

\$ Socio-economic grouping is based on highest ranking parents. Lowest grouping includes those whose parents' occupation was semi-routine and routine and those who never worked or were long-term unemployed (see methods for more details). §Questions refer to childhood before menarche/puberty (see methods for more details). $* *<0.01$, $* * *<0.001$.

menarche earlier. For males, LR analysis identified earlier pubertal onset with more recent year of birth (i.e. $<25$ years old at survey); being gay or bisexual; and having had parents categorised in the poorer socio-economic group. Paper based and online data both showed associations with pubertal onset consistent with LR results but only age (paper-based) and sexuality (online) reached significance individually (table 3).
Tables 4 (female) and 5 (male) explore associations between early adolescent risk behaviours and age of pubertal onset. For females, having had sex before age 16 years and having drunk alcohol or been drunk before age 14 years were all associated with earlier menarche in online, paper-based and combined (stratified) analyses (table 4). In addition however, having had unprotected sex (i.e. without any contraception) under 16 years and

Table 2: Demographic and pre-pubertal predictors of earlier menarche in females

\begin{tabular}{|c|c|c|c|c|c|c|c|c|c|c|c|c|}
\hline & & \multicolumn{7}{|c|}{ Age at menarche (years) } & \multicolumn{4}{|c|}{ Total $^{+}$} \\
\hline & & \multicolumn{4}{|c|}{ Online (\%) } & \multicolumn{3}{|c|}{ Paper (\%) } & \multirow[b]{2}{*}{$\mathbf{P}$} & \multirow[b]{2}{*}{ AOR } & \multirow[b]{2}{*}{$95 \% \mathrm{Cl}$} & \multirow[b]{2}{*}{$\mathbf{P}$} \\
\hline & & $\mathbf{n}$ & $\leq 12$ & $\geq 13$ & $\mathbf{P}$ & $\mathbf{n}$ & $\leq 12$ & $\geq 13$ & & & & \\
\hline Age at & $16-24$ years & 345 & 44.3 & 39.7 & 0.179 & 495 & 84.6 & 86.3 & 0.559 & ns & & \\
\hline survey & $\geq 25$ years & 477 & 55.7 & 60.3 & & 83 & 15.4 & 13.7 & & & & \\
\hline \multirow[t]{2}{*}{ Ethnicity } & white & 792 & 95.8 & 97.3 & 0.243 & 547 & 93.8 & 95.4 & 0.387 & ns & & \\
\hline & other & 28 & 4.2 & 2.7 & & 30 & 6.2 & 4.6 & & & & \\
\hline \multirow[t]{2}{*}{ Sexuality } & heterosexual & 749 & 91.5 & 91.9 & 0.825 & 527 & 95.8 & 95.3 & 0.786 & ns & & \\
\hline & lesbian/bisexual & 68 & 8.5 & 8.1 & & 25 & 4.2 & 4.7 & & & & \\
\hline Number of & $\leq 1$ & 506 & 59.6 & 63.5 & 0.251 & 443 & 72.7 & 79.2 & 0.071 & Ref & & \\
\hline childhood illnesses§ & $\geq 2$ & 316 & 40.4 & 36.5 & & 135 & 27.3 & 20.8 & & 1.87 & $(0.98-3.57)$ & * \\
\hline \multirow[t]{2}{*}{ Overweight $\$$} & no & 704 & 83.2 & 88.3 & $*$ & 493 & 78.8 & 89.7 & $* * *$ & Ref & & \\
\hline & yes & 117 & 16.8 & 11.7 & & 84 & 21.2 & 10.3 & & 1.89 & $(1.36-2.63)$ & $* * *$ \\
\hline Father at & no & 160 & 18.7 & 21.4 & 0.340 & 119 & 22.1 & 21.4 & 0.838 & ns & & \\
\hline home§ & yes & 640 & 81.3 & 78.6 & & 430 & 77.9 & 78.6 & & & & \\
\hline Days off & $\leq 10$ & 672 & 78.0 & 86.7 & 0.712 & 479 & 73.5 & 84.3 & 0.144 & ns & & \\
\hline school per year§ & $\geq \mathrm{II}$ & 142 & 22.0 & 13.3 & & 91 & 26.5 & 15.7 & & & & \\
\hline In poorest socio- & no & 501 & 61.2 & 66.8 & 0.105 & 415 & 75.2 & 79.8 & 0.213 & Ref & & \\
\hline economic grouping\$§ & yes & 282 & 38.8 & 33.2 & & 117 & 24.8 & 20.2 & & 1.32 & $(1.03-1.71)$ & * \\
\hline
\end{tabular}

\$Socio-economic grouping is based on highest ranking parents. Lowest grouping includes those whose parents' occupation was semi-routine and routine and those who never worked or were long-term unemployed (see methods for more details). §Questions refer to childhood before menarche (see methods for more details). $*<0.05, * *<0.01$, $* * *<0.00 \mathrm{I}$, ns $=$ not significant. ${ }^{+}$Logistic regression analysis utilised all variables in table as well as data source (i.e. online or paper), $\mathrm{AOR}=$ Adjusted Odds Ratio, $\mathrm{Cl}=$ Confidence Intervals. 
Table 3: Demographic and pre-pubertal predictors of earlier puberty onset in males

\begin{tabular}{|c|c|c|c|c|c|c|c|c|c|c|c|c|}
\hline & & \multicolumn{8}{|c|}{ Age of puberty (years) } & \multicolumn{3}{|c|}{ Total $^{+}$} \\
\hline & & \multicolumn{4}{|c|}{ Online (\%) } & \multicolumn{3}{|c|}{ Paper (\%) } & \multirow[b]{2}{*}{$\mathbf{P}$} & \multirow[b]{2}{*}{ AOR } & \multirow[b]{2}{*}{$95 \% \mathrm{Cl}$} & \multirow[b]{2}{*}{$\mathbf{P}$} \\
\hline & & $\mathbf{n}$ & $\leq \mathrm{II}$ & $\geq 12$ & $\mathbf{P}$ & $\mathbf{n}$ & $\leq \mathrm{II}$ & $\geq 12$ & & & & \\
\hline Age at & 16-24 years & 143 & 51.6 & 45.1 & 0.271 & 363 & 94.7 & 88.9 & $*$ & Ref & & \\
\hline survey & $\geq 25$ years & 151 & 48.4 & 54.9 & & 32 & 5.3 & 11.1 & & 0.64 & $(0.44-0.94)$ & $*$ \\
\hline \multirow[t]{2}{*}{ Ethnicity } & white & 274 & 93.8 & 93.2 & 0.834 & 376 & 95.1 & 95.7 & 0.776 & & & \\
\hline & other & 19 & 6.2 & 6.8 & & 18 & 4.9 & 4.3 & & ns & & \\
\hline \multirow[t]{2}{*}{ Sexuality } & heterosexual & 207 & 65.8 & 76.5 & $*$ & 363 & 91.7 & 92.6 & 0.744 & & & \\
\hline & gay/bisexual & 86 & 34.2 & 23.5 & & 31 & 8.3 & 7.4 & & 1.97 & $(1.25-3.11)$ & ** \\
\hline Number of childhood & $\leq \mathrm{I}$ & 190 & 64.0 & 65.4 & 0.797 & 315 & 80.6 & 78.8 & 0.666 & ns & & \\
\hline illnesses§ & $\geq 2$ & 104 & 36.0 & 34.6 & & 80 & 19.4 & 21.2 & & & & \\
\hline \multirow{2}{*}{ Overweight $\S$} & no & 270 & 91.3 & 92.5 & 0.714 & 347 & 85.9 & 91.0 & 0.116 & ns & & \\
\hline & yes & 24 & 8.7 & 7.5 & & 46 & 14.1 & 9.0 & & & & \\
\hline Father at & no & 54 & 15.8 & 22.9 & 0.129 & 89 & 25.5 & 21.5 & 0.365 & ns & & \\
\hline home§ & yes & 229 & 84.2 & 77.1 & & 288 & 74.5 & 78.5 & & & & \\
\hline Days off & $\leq 10$ & 221 & 80.5 & 82.6 & 0.316 & 316 & 81.0 & 79.5 & 0.105 & ns & & \\
\hline school per year§ & $\geq 11$ & 72 & 19.5 & 17.4 & & 77 & 19.0 & 20.5 & & & & \\
\hline In poorest socio- & no & 189 & 65.8 & 72.4 & 0.243 & 293 & 74.6 & 82.3 & 0.07 & Ref & & \\
\hline economic grouping $\$ \S$ & yes & 86 & 34.2 & 27.6 & & 81 & 25.4 & 17.7 & & 1.59 & $(1.09-2.31)$ & $*$ \\
\hline
\end{tabular}

\$Socio-economic grouping is based on highest ranking parents. Lowest grouping includes those whose parents' occupation was semi-routine and routine and those who never worked or were long-term unemployed (see methods for more details). \$Questions refer to childhood before the age of I 2 years (see methods for more details). $*<0.05, * *<0.0 \mathrm{I}, * * *<0.00 \mathrm{I}, \mathrm{ns}=$ not significant. ${ }^{+}$Logistic regression analysis utilised all variables in table as well as data source (i.e. online or paper), $\mathrm{AOR}=$ Adjusted Odds Ratio, $\mathrm{Cl}=$ Confidence Intervals.

Table 4: Associations between adolescent risk behaviours and age of menarche in females

\begin{tabular}{|c|c|c|c|c|c|c|c|c|c|c|c|c|c|}
\hline & & \multicolumn{12}{|c|}{ Age of menarche (years) } \\
\hline & & \multicolumn{4}{|c|}{ Online (\%) } & \multicolumn{4}{|c|}{ Paper (\%) } & \multicolumn{4}{|c|}{ Total (\%) } \\
\hline & & $\mathbf{n}$ & $\leq \mathbf{1 2}$ & $\geq 13$ & $\mathbf{P}$ & $\mathbf{n}$ & $\leq 12$ & $\geq 13$ & $\mathbf{P}$ & $\mathbf{n}$ & $\leq \mathbf{1 2}$ & $\geq 13$ & $\mathbf{P}^{\neq}$ \\
\hline & drank alcohol & 812 & 46.9 & 38.5 & $*$ & 572 & 59.8 & 47.4 & $* *$ & 1384 & 51.5 & 42.6 & $* * *$ \\
\hline Before age & was drunk & 820 & 25.7 & 19.0 & $*$ & 572 & 36.2 & 26.7 & $*$ & 1392 & 29.4 & 22.5 & $* *$ \\
\hline \multirow[t]{3}{*}{14 years, } & took drugs & 806 & 5.9 & 3.3 & 0.071 & 559 & 8.7 & 2.9 & $* *$ & 1365 & 6.9 & 3.1 & $* *$ \\
\hline & smoked & 822 & 29.0 & 22.6 & $*$ & 570 & 29.3 & 22.4 & 0.065 & 1392 & 29.1 & 22.5 & ** \\
\hline & had sex & 820 & 32.8 & 22.4 & $* * *$ & 554 & 35.0 & 23.1 & $*$ & 1374 & 33.5 & 22.7 & $* * *$ \\
\hline \multirow{3}{*}{$\begin{array}{l}\text { Before age } \\
16 \text { years, }\end{array}$} & had unprotected sex & 820 & 13.4 & 11.0 & 0.286 & 563 & 13.5 & 7.3 & $*$ & 1383 & 13.4 & 9.3 & * \\
\hline & first pregnant & 822 & 1.0 & 1.0 & 1.000 & 578 & 1.3 & 0.6 & 0.341 & 1400 & I.I & 0.8 & 0.763 \\
\hline & had an abortion & 822 & 12.4 & 13.1 & 0.754 & 578 & 7.9 & 6.6 & 0.529 & 1400 & 10.8 & 10.1 & 0.986 \\
\hline \multicolumn{2}{|c|}{$\int$ Took drugs/alcohol at first sex } & 785 & 28.4 & 28.4 & 0.991 & 509 & 28.8 & 20.6 & $*$ & 1294 & 28.5 & 25.0 & 0.234 \\
\hline \$Typical & aggression & 142 & 19.0 & 15.7 & 0.324 & 93 & 16.0 & 17.6 & 0.428 & 235 & 18.0 & 16.6 & 0.782 \\
\hline reactions to & ignoring people & 226 & 25.9 & 29.5 & & 153 & 31.0 & 25.9 & & 379 & 27.6 & 27.9 & \\
\hline emotional upset & moodiness & 449 & 55.1 & 54.8 & & 303 & 53.1 & 56.5 & & 752 & 54.4 & 55.6 & \\
\hline \multirow[t]{2}{*}{ Bullying } & victim & 820 & 63.4 & 56.1 & $*$ & 573 & 50.0 & 46.1 & 0.362 & 1393 & 58.6 & 51.5 & $*$ \\
\hline & perpetrator & 822 & 18.0 & 19.2 & 0.654 & 564 & 17.4 & 12.4 & 0.094 & 1386 & 17.8 & 16.1 & 0.580 \\
\hline §Frequency & Never & 607 & 71.6 & 76.8 & 0.392 & 448 & 74.0 & 80.2 & 0.108 & 1055 & 72.5 & 78.4 & * \\
\hline of & $<$ l/year & 138 & 19.3 & 14.4 & & 64 & 12.8 & 10.0 & & 202 & 17.0 & 12.4 & \\
\hline involvement & circa I/year & 47 & 6.4 & 5.1 & & 38 & 7.9 & 5.7 & & 85 & 6.9 & 5.4 & \\
\hline in fighting & $>$ I/year & 26 & 2.7 & 3.7 & & 26 & 5.3 & 4.0 & & 52 & 3.6 & 3.8 & \\
\hline Time off & $\leq 10$ days & 672 & 78.0 & 86.7 & $* *$ & 450 & 73.5 & 84.3 & $* *$ & 1122 & 76.4 & 85.6 & $* * *$ \\
\hline school/year & $\geq$ II days & 144 & 22.0 & 13.3 & & 112 & 26.5 & 15.7 & & 256 & 23.6 & 14.4 & \\
\hline
\end{tabular}

Questions not relating to a specific age refer to adolescence (here measured as the time period post menarche). $*<0.05, * *<0.0 \mathrm{I}, * * *<0.00 \mathrm{I}, \mathrm{ns}=$ not significant. \$Respondents could select only one answer. \$Analyses utilised chi squared for a trend. $¥$ Combined analyses on dichotomised variables utilised a stratified chi-squared design. JAnalyses limited to those individuals who reported ever having had sex. 
Table 5: Associations between adolescent risk behaviours and age of pubertal onset in males

\begin{tabular}{|c|c|c|c|c|c|c|c|c|c|c|c|c|c|}
\hline & & \multicolumn{12}{|c|}{ Estimated age of pubertal onset (years) } \\
\hline & & \multicolumn{4}{|c|}{ Online (\%) } & \multicolumn{4}{|c|}{ Paper (\%) } & \multicolumn{4}{|c|}{ Total (\%) } \\
\hline & & $\mathbf{n}$ & $\leq \mathbf{I I}$ & $\geq 12$ & $\mathbf{P}$ & $\mathbf{n}$ & $\leq \mathrm{II}$ & $\geq 12$ & $\mathbf{P}$ & $\mathbf{n}$ & $\leq \mathrm{II}$ & $\geq 12$ & $\mathbf{P}^{¥}$ \\
\hline & drank alcohol & 294 & 55.3 & 33.1 & $* * *$ & 392 & 56.6 & 41.7 & $* *$ & 686 & 56.0 & 38.1 & **** \\
\hline Before age & was drunk & 294 & 26.7 & 13.5 & $* *$ & 390 & 38.9 & 26.2 & $* *$ & 684 & 33.5 & 20.9 & $* * *$ \\
\hline \multirow[t]{2}{*}{14 years, } & took drugs & 284 & 11.7 & 2.3 & $* *$ & 393 & 10.7 & 5.3 & 0.050 & 677 & 11.1 & 4.1 & $* *$ \\
\hline & smoked & 293 & 28.1 & 15.8 & $*$ & 392 & 28.9 & 14.9 & $* * *$ & 685 & 28.6 & 15.3 & $* * *$ \\
\hline Before age & had sex & 292 & 33.5 & 13.0 & $* * *$ & 392 & 40.4 & 19.0 & $* * *$ & 684 & 37.4 & 16.6 & $* * *$ \\
\hline 16 years, & had unprotected sex & 292 & 23.3 & 5.3 & $* * *$ & 390 & 17.7 & 8.0 & $* *$ & 682 & 20.2 & 6.9 & **** \\
\hline \multicolumn{2}{|c|}{$\int$ Took drugs/alcohol at first sex } & 256 & 29.2 & 20.5 & 0.115 & 354 & 36.2 & 37.3 & 0.818 & 610 & 33.1 & 30.6 & 0.494 \\
\hline \$Typical & aggression & 68 & 28.0 & 17.4 & 0.070 & 184 & 56.4 & 41.8 & $*$ & 252 & 43.5 & 31.4 & ** \\
\hline reactions to & ignoring people & 147 & 49.1 & 51.5 & & 136 & 30.8 & 42.9 & & 283 & 39.0 & 46.6 & \\
\hline emotional upset & moodiness & 78 & 23.0 & 31.1 & & 52 & 12.8 & 15.3 & & 130 & 17.4 & 22.0 & \\
\hline \multirow[t]{2}{*}{ Bullying } & victim & 294 & 70.2 & 69.2 & 0.851 & 392 & 38.2 & 46.8 & 0.086 & 686 & 52.3 & 56.1 & 0.254 \\
\hline & perpetrator & 294 & 40.4 & 33.8 & 0.249 & 388 & 44.1 & 39.2 & 0.337 & 682 & 42.4 & 37.0 & 0.162 \\
\hline$\S$ Frequency & Never & 103 & 30.8 & 40.6 & $*$ & 128 & 27.2 & 38.3 & $* *$ & 231 & 28.8 & 39.3 & $* *$ \\
\hline of & $<$ l/year & 91 & 29.6 & 33.1 & & 95 & 23.3 & 25.0 & & 186 & 26.0 & 28.3 & \\
\hline involvement & circa I/year & 61 & 23.3 & 18.0 & & 104 & 29.6 & 22.9 & & 165 & 26.8 & 20.9 & \\
\hline in fighting & $>$ I/year & 37 & 16.4 & 8.3 & & 67 & 19.9 & 13.8 & & 104 & 18.4 & 11.5 & \\
\hline Time off & $\leq 10$ days & 237 & 80.5 & 82.6 & 0.651 & 309 & 81.0 & 79.5 & 0.704 & 546 & 80.8 & 80.8 & 0.924 \\
\hline school/year & $\geq \mathrm{II}$ days & 54 & 19.5 & 17.4 & & 76 & 19.0 & 20.5 & & 130 & 19.2 & 19.2 & \\
\hline
\end{tabular}

Questions not relating to a specific age refer to adolescence (here measured as the time period post I2 years). $*<0.05$, $* *<0.01$, $* * *<0.00 \mathrm{I}, \mathrm{ns}=\mathrm{not}$ significant. \$Respondents could select only one answer. §Analyses utilised chi squared for a trend. $¥$ Combined analyses on dichotomised variables utilised a stratified chi-squared design.

Analyses limited to those individuals who reported ever having had sex.

having taken an illicit drug or smoked under 14 years were significant in the combined analyses. For females, the number of days off school per year (post menarche) was higher in those having earlier puberty in both online and paper-based surveys as well as in the combined analysis. Finally, being bullied and increased frequency of fighting both showed significant relationships with earlier puberty in combined analyses. For males, drinking alcohol, having been drunk, taking drugs and smoking under age 14 years were all significantly associated with earlier puberty in the combined analysis with all (except taking drugs) also being significant in both online and paper-based surveys. Having had sex and unprotected sex under 16 years were also significantly related to earlier puberty in individual and combined data sets (table 5). Males with earlier pubertal markers reported higher frequencies of adolescent fighting and were more likely to respond to emotional upset with aggression; although the latter narrowly failed to reach significance in the online survey.

Logistic regression was used to identify the independent relationships between pubertal onset and risk behaviours (sexual risk taking, alcohol, smoking and drug use), time off school, bullying, and typical response to being upset. Thus for each behaviour, figure 1 (female) and figure 2 (male) show the significant adjusted odds ratios (AORs; relating to earlier puberty) after controlling for age at time of survey, socio-economic grouping, ethnicity, sexuality, and data collection method (same categorisation of varia- bles as table 1). Here, pubertal onset in females (i.e. menarche) was independently related to drinking, having been drunk, smoking and drug use under 14 years; having had sex and unprotected sex under 16 years; being bullied; and having more time off school per year. For males (figure 2), earlier puberty independently significantly predicted the same factors (as females) with the exception of being bullied and time off school but with the addition of frequency of fighting and aggressively responding to emotional upset. For all substance use and especially sexual behaviours the increases in odds relating to earlier puberty were greater in males than females (e.g. had sex under 16 years; AOR $+/-95 \%$ confidence intervals, males, 3.21, 2.19-4.69; females, 1.72, 1.34-2.21).

\section{Discussion}

Two different populations were examined in order to independently explore pre and peri-pubertal factors predictive of earlier puberty and to understand how age at puberty subsequently relates to health and adoption of health damaging behaviours. Importantly, having two different samples provided some measure of how consistent relationships between pubertal onset and risk behaviours are across two independent populations. Both online and paper-based surveys were limited by opportunistic sampling and, combined with completion rates of around $60 \%$, raise the probability of self-selection and sample bias. However, analyses only examined relationships between variables describing any individual and did not 


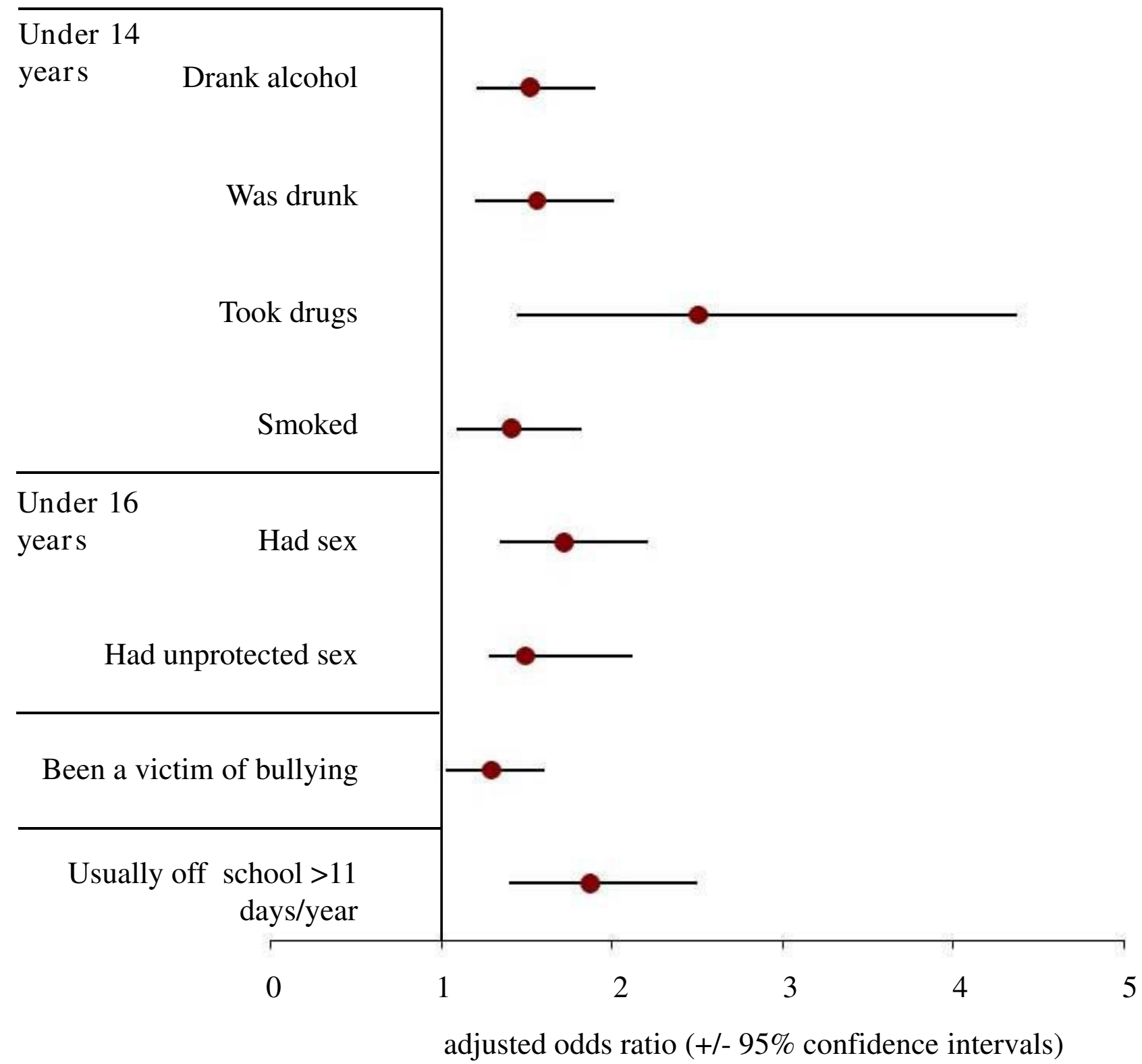

Figure I

Effects of menarche $\leq \mathbf{I} 2$ years on odds of involvement in different early adolescent behaviours. Analysis employed backwards conditional logistic regression to examine behaviour undertaken before ages 14 and 16 years. Emotional response to upset and frequency of fighting were also analysed using multinomial logistic regression but identified no significant relationships with age of menarche and therefore are not shown. For all analyses, other than pubertal onset, independent variables included all key demographics measured: age at time of survey, socio-economic grouping, ethnicity, sexuality, and data collection method. The categories used for these variables are as described in table $\mathrm{I}$.

attempt to extrapolate to wider populations. The study also employed retrospective proxy measurements for puberty. Menarche is a well established correlate with puberty for girls. Although retrospective studies can be prone to errors in data recollection our median menarcheal age (13.0 years) is in line with other studies examining age at menarche in the UK (i.e. median age 12 years, 11 months) [4]. For males, physiological measures, such as spermarche and facial hair growth, have been used as markers of puberty [38]. However, like other studies 


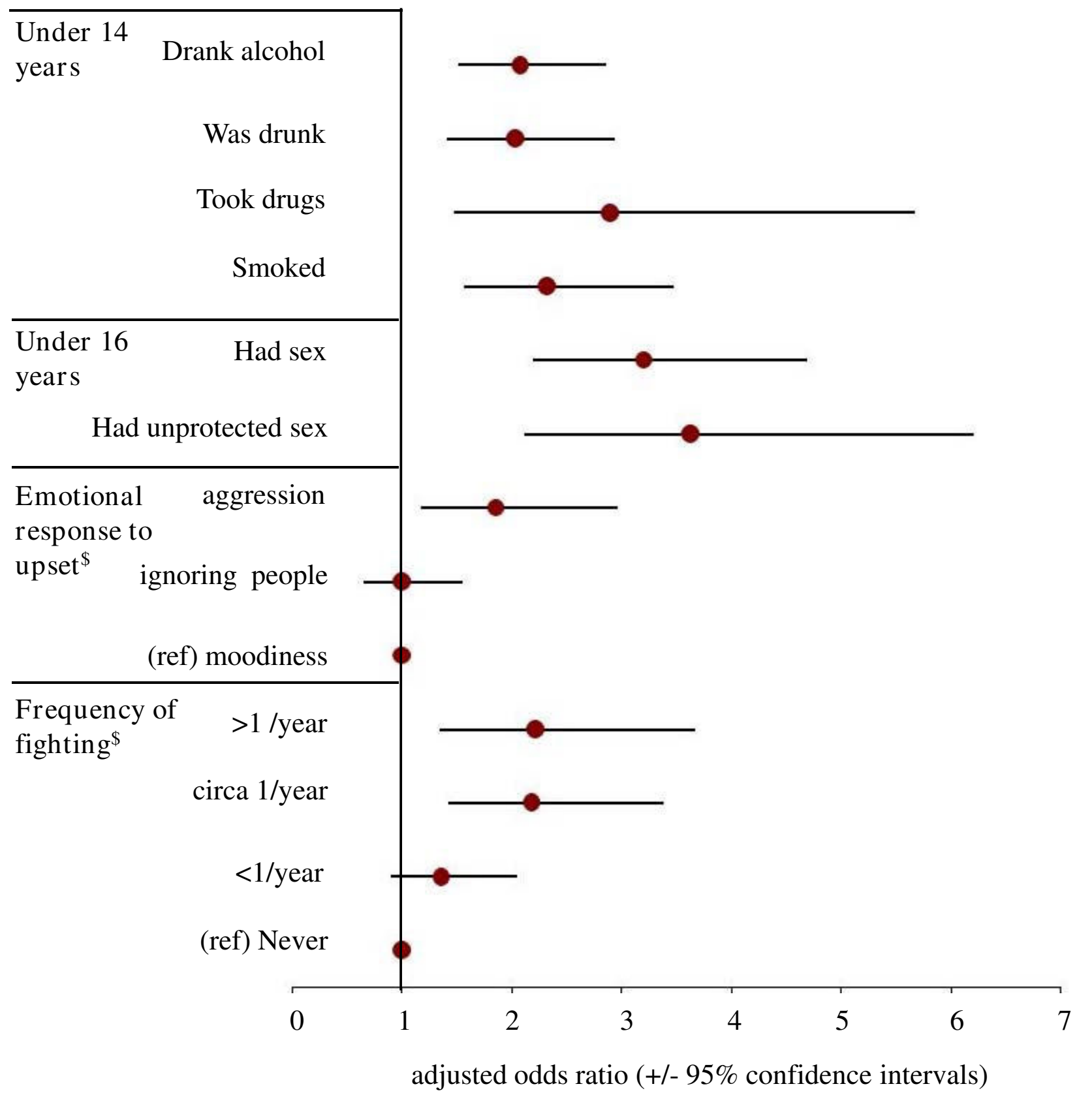

Figure 2

Effects of pubertal onset $\leq$ I I years on odds of involvement in different early adolescent behaviours. Analysis employed backwards conditional logistic regression to examine behaviour undertaken before ages 14 and 16 years. \$For emotional response to upset and frequency of fighting multinomial logistic regression was employed. Reference categories are only shown for multinomial analyses. For all analyses, other than pubertal onset, independent variables included all key demographics measured: age at time of survey, socio-economic grouping, ethnicity, sexuality, and data collection method. The categories used for these variables are as described in table I.

[15] we employed a range of behavioural markers as proxies for pubertal onset. Importantly, our proxy measures produced a median age of 11.0 years and while there are no comparable data from the UK this is consistent with studies elsewhere (USA; range: 10-12 years) [3]. Retro- spective design also risked problems with passage of time confounding recall of other variables (e.g. self-assessed peri-pubertal weight, family structure, personal illness and substance use and sexual behaviours) and subsequently their relationships with pubertal onset. However, 
although our online and paper based samples differed significantly in age structure (table 1) both independently identified similar relationships between weight and early puberty in girls (table 2) and early pubertal onset and alcohol and sex-related risk behaviours in both sexes (tables 4 and 5). Finally, by exploring a range of factors potentially relating to early puberty and subsequent risk behaviours, there is a risk of Type I errors [40]. However, we limited independent variables to key demographics and those factors previously identified as linked with age of puberty in other studies. Importantly, with this study being an initial examination of potential public health consequences relating to earlier pubertal onset we opted not to include family-wise alpha corrections which would increase risks of type II errors $[41,42]$.

The implications of a reducing pubertal age for public health, and in particular for sexual health, substance use and anti-social behaviour, may be substantial. These initial results are insufficient to establish population level effects but at least suggest that alcohol consumption, having been drunk, drug use and smoking under 14 years may be associated with earlier puberty in both sexes. Thus, the proportion of females drunk under 14 years increases from $22.5 \%$ in those with menarche $\geq 13$ years to $29.4 \%$ in those with menarche $\leq 12$ years (table 4 ). Early onset alcohol consumption is linked with adolescent binge drinking [43] and an increased chance of alcohol dependence in later life $[11,44]$. Our results also suggest a relationship for both sexes between sexual risk-taking and earlier markers of puberty. Thus in boys, the odds of having had sex or unprotected sex under 16 years were respectively 3.21 and 3.63 times higher in those experiencing puberty earlier (figure 2). We could not examine what mechanisms might link earlier puberty with either adolescent substance use or sexual risk-taking. However, the temporal division between physical development and social maturity, social responsibility and independence may be a contributory factor $[1,39]$. Moreover, such divisions can be compounded when formal education on risk-taking (e.g. sexual or substance related) or even informal discussions with parents are delayed as public bodies and parents fail to recognise earlier pubertal onset. Consequently, adolescents and especially those passing through puberty relatively early may be ill-equipped to deal with emerging sexual and other exploratory urges.

Males and females differed across a number of pre and peri-pubertal factors predictive of earlier puberty and subsequent, associated risk behaviours (tables 4 and 5). For males, analyses identified associations between earlier puberty and both increased frequency of early adolescent fighting and likelihood of responding to emotional upset with aggression (figure 2). Such results at least suggest a hypothesis that earlier puberty is a contributor to, or a risk factor for, a greater propensity for violence. Previous studies have shown similar links between earlier maturation and aggression in girls and boys $[45,46]$ although our results found no relationship between female aggression or violence and pubertal timing; once demographics had been corrected for (table 4, figure 1). For females earlier puberty was strongly associated with self-assessed weight prior to menarche (table 2). Despite retrospective reporting of weight problems being potentially prone to recall issues and variations in social norms, our results were largely consistent with studies examining pubertal onset and weight elsewhere [34]. Overall $18.4 \%$ of those with menarche $\leq 12$ years reported being overweight compared to only $11.0 \%$ of those who reported menarche at $\geq 13$ years. This finding is consistent with studies in other countries examining body mass index and pubertal timing [34]. However, in England levels of obese and overweight children are already high (ages 2-10 years, overweight, $13 \%$ males, $14 \%$ females; obese, $16 \%$ males, $14 \%$ females) [30] and are forecast to increase [47]. While the direct effects of being overweight on immediate (e.g. body dissatisfaction disorders) [23] and long term health (e.g. higher risk of mortality) [48] have been considered in some detail, the potential for weight to affect adolescent sexual health, substance use and violence have received little attention.

Our results suggest that the occurrence (tables 2 and 3 ) of early puberty should be explored as a potential factor influencing adolescent behaviour especially in deprived communities and as a possible contributor to health inequalities. For both sexes those whose parents were classified in the poorest socio-economic group were more likely to experience puberty earlier (tables 2 and 3). While little work has explored differences in pubertal onset associated with poverty, national surveys identify children (aged 1011 years) living in disadvantaged areas as more likely to be overweight or obese [49]. Thus, weight may be one factor linking early puberty and deprivation. However, in males we found no association between weight and pubertal onset (table 3 ) while in females, the association between weight and pubertal timing remained even after correcting for poverty (table 2). Teenage pregnancy [27], sexually transmitted infections [28], illicit drug use [29], smoking [30], and underage hospital admissions for alcohol use and violence $[31,32]$ are all reported at higher levels in adolescents resident in poorer communities. In light of the retrospective nature of this study, results here are insufficient to quantify any contribution that earlier puberty may be making to the relationship between these major public health issues and pubertal onset. However, our results suggest an urgent need to examine how diet and deprivation are affecting maturation and behavioural development in children and what measures can be put in place to reduce associated harms. 


\section{Conclusion}

The opportunistic samples examined here were neither of sufficient size or demonstrably representative to extrapolate to broader populations. However, two different samples both provided some evidence of associations between early pubertal onset and major public health challenges facing a youth that physically develop at a substantially greater rate than 150 years ago [6]. Thus, with little consideration of the health consequences, societal developments have created an extended period of adolescence where teenagers physically mature long before they are regarded as sufficiently socially and emotionally capable to function as adults [1]. In fact, social and emotional development may even be deliberately obstructed as preand peri-pubescent individuals are denied sexual and other knowledge ostensibly to protect their innocence. Internationally the sexual risk-taking, substance use and other anti-social challenges presented by adolescents are an increasing threat to public health [50]. Our results add to a growing literature $[11,12]$ that suggests these challenges are associated with a decreasing age at which many youths now experience puberty. Whether links between earlier puberty and sexual risk-taking, substance use and violent behaviours result from juvenile coping strategies, ill-informed exploratory and rebellious tendencies or some other mediator requires further examination. However, health strategies should consider how epidemic levels of child obesity are contributing to a divergence between physical and social puberty which may subsequently affect youth risk behaviours. Moreover, a general reduction in age of pubertal onset [6] should support public health calls for earlier substance use and sexual health education in schools, and should inform campaigns to encourage related pre-pubescent discussion between parents and children. Finally, a greater insight into what is shortening childhood may provide new mechanisms to tackle health inequalities and reduce the harms from teenage pregnancy, substance misuse and violent behaviour which fall most heavily on disadvantaged communities.

\section{Competing interests}

The authors declare that they have no competing interests.

\section{Authors' contributions}

JD designed, developed the study, analysed the data and wrote the manuscript. MAB designed and developed the study, contributed to the analysis and co-wrote the manuscript. All Authors have read and approved the final manuscript.

\section{Acknowledgements}

We would like to thank Hayley Jones, Fintan Shevlin and all other assistants for their help with data collection and data entry. We would also like to thank all those who commented on the draft questionnaires and who participated in the pilot or full study. Special thanks are due to Karen Hughes and Clare Perkins for their comments on the draft manuscript and John
Ashton for discussion on the potential for puberty to influence public health. Funding for this work was provided from Centre for Public Health, Liverpool John Moores University. Finally, we are grateful to the Steve Shoptaw, Muideen Bakare and the editorial team at BMC Public Health for comments on an earlier version of this paper.

\section{References}

I. Bellis MA, Downing J, Ashton JA: Adults at I 2? Trends in puberty and their public health consequences. I Epidemiol Community Health 2006, 60:910-911.

2. De Muinck Keizer-Schrama SM, Mull D: Trends in pubertal development in Europe. Hum Reprod Update 2001, 7:287-29I.

3. Herman-Giddens ME: Recent data on pubertal milestones in United States children: the secular trend toward earlier development. Int J Androl 2006, 29:24I-246.

4. Whincup PH, Gilg JA, Odoki K, Taylor SJC, Cook DG: Age of menarche in contemporary British teenagers: survey of girls born between 1982 and 1986. BMJ 2001, 322:1095-1096.

5. Anderson SE, Must A: Interpreting the continued decline in the average age at menarche, results from two nationally representative surveys of US girls studies 10 years apart. J Pediatr 2005, I 47:753-760.

6. Bodzsar EB, Susanne C, Eds: Secular growth changes in Europe. Budapest, Hungary Budapest: Eotvos University Press; 1998.

7. Bogaert AF: Age at puberty and father absence in a national probability sample. J Adolesc 2005, 28:54I-546.

8. Gluckman PD, Hanson MA: Changing times: The evolution of puberty. Mol Cell Endocrinol 2006, 254-255:26-3I.

9. Zehr JL, Culbert KM, Sisk CL, Klump KL: An association of early puberty with disordered eating and anxiety in a population of undergraduate women and men. Horm Behav 2007, 52:427-435.

10. Kaltiala-Heino R, Rimpela M, Rissanen A, Rantanen P: Early puberty and early sexual activity are associated with bulimic-type eating pathology in middle Adolescence. J Adolesc Health 200 I, 28:346-352.

II. Costello EJ, Sung M, Worthman C, Angold A: Pubertal maturation and the development of alcohol use and abuse. Drug Alcohol Depend 2007, 88S:S50-S59.

12. Westling E, Andrews JA, Hampson SE, Peterson M: Pubertal timing and substance use: the effects of gender, parental monitoring and deviant peers. J Adolesc Health 2008, 42:555-563.

13. Zimmer-Gembeck MJ, Collins WA: Gender, mature appearance, alcohol use, and dating as correlates of sexual partner accumulation from ages 16-26 years. J Adolesc Health 2008, 42:564-572.

14. Johnson AM, Mercer CH, Erens B, Copas AJ, McManus S, Wellings K, Fenton KA, Korovessis C, Macdowall W, Nanchahal K, Purdon S, Field J: Sexual behaviour in Britain: partnerships, practices, and HIV risk behaviours. Lancet 200I, 358: I835-42.

15. Edgardh K: Sexual behaviour and early coitarche in a national sample of I7-year old Swedish boys. Acta Paediatr 2002, 91:985-991.

16. Felson RB, Haynie DL: Pubertal Development, social factors, and delinquency among adolescent boys. Criminology 2002, 40:967-988.

17. Fenton KA, Lowndes CM: Recent trends in the epidemiology of sexually transmitted infections in the European Union. Sex Transm Inf 2004, 80:255-263.

18. ESSTI: Sexually Transmitted Infections in Europe; annual report No. 3 London: Health Protection Agency; 2008.

19. Hibell B, Guttormsson U, Ahlströhm S, Balakireva O, Bjarnason T, Kokkevi A, Kraus L: The 2007 ESPAD Report: substance use among students in 35 European countries Stockholm: ESPAD; 2009.

20. Goodson P, Evans A, Edmundson E: Female adolescents and onset of sexual intercourse: a theory-based review of research from 1984 to 1994. J Adolesc Health 1997, 21:147-156.

21. Kaltiala-Heino R, Kosunen E, Rimpela M: Pubertal timing, sexual behaviour and self-reported depression in middle adolescence. J Adolesc 2003, 26:531-545.

22. Michaud PA, Suris JC, Deppen A: Gender-related psychological and behavioural correlates of pubertal timing in a national sample of Swiss adolescents. Mol Cell Endocrinol 2006, 254255: $172-178$. 
23. Berger U, Weitkamp K, Strauss B: Weight Limits, estimations of future BMI, subjective pubertal timing and physical appearance comparisons among adolescent girls as precursors of disturbed eating behaviour in a community sample. Eur Eat Disorders Rev 2009, 17:128-136.

24. Unicef: Child poverty in perspective: An overview of child well-being in rich countries. Innocenti report card 7 Florence: Unicef; 2007.

25. Unicef: A league table of teenage births in rich nations. Innocenti report card 3 Florence: Unicef; 2001.

26. Christie D, Viner R: Adolescent development. BMJ 2005, 330:30I-304.

27. Bellis MA, Morleo M, Tocque K, Dedman D, Phillips-Howard PA, Perkins C, Jones L: Contributions of alcohol use to teenage pregnancy: an examination of geographical and evidence based associations Liverpool: North West Public Health Observatory, Centre for Public Health, Liverpool John Moores University; 2009.

28. Holtgrave DR, Crosby RA: Social capital, poverty, and income inequality as predictors of gonorrhoea, syphilis, chlamydia and AIDS case rates in the United States. Sex Transm Inf 2003, 79:62-64.

29. Anderson P: Global use of alcohol, drugs and tobacco. Drug Alcohol Rev 2006, 25:489-502.

30. Chaudhury M, Falaschetti E, Fuller E, Mackenzie H, Mindell J, Nicholson S, Pickup D, Roth M, Scholes S, Tabassaum F, Thompson J, Wardle H: Health survey for England 2007. Healthy lifestyles: knowledge, attitudes and behaviour Volume I. London: The NHS Information Centre; 2008.

31. Bellis MA, Hughes K, Anderson Z, Tocque K, Hughes S: Contribution of violence to health inequalities in England: demographics and trends in emergency hospital admissions for assault. J Epidemiol Community Health 2008, 62: 1064-107I.

32. Jones L, Bellis MA, Dedman D, Sumnall H, Tocque K: Alcoholattributable fractions for England; alcohol-attributable mortality and hospital admissions. Liverpool: Centre for Public Health, Liverpool John Moores University; 2008.

33. Bogaert AF, Friesen C: Sexual orientation and height, weight, and age of puberty: new tests from a British national probability sample. Biol Psychol 2002, 59:135-145.

34. Anderson SE, Gerard MS, Dallal E, Must A: Relative weight and race influence average Age at menarche: results from two nationally representative surveys of US girls studied 25 years apart. Pediatrics 2003, I I I:844-850.

35. Mul D, Oostdijk W, Drop SLS: Early puberty in adopted children. Horm Res 2002, 57:1-9.

36. Office for National Statistics: Standard occupational classification 2000; structure and description of unit groups Volume I. London: the Stationary Office; 2000.

37. Office for National Statistics: Standard occupational classification 2000; the coding index Volume 2. London: The Stationary Office; 2000.

38. Coleman L, Coleman J: The measurement of puberty: a review. J Adolesc 2002, 25:535-550.

39. Gluckman PD, Hanson MA: Evolution, development and timing of puberty. Trends Endocrinol Metab 2006, 17:7-12.

40. Perneger TV: What's wrong with Bonferroni adjustments. $B M J$ 1998, 3 16: | 236-1238.

4I. O'Keefe DJ: Colloquy: Should familywise alpha be adjusted? Against familywise alpha adjustment. Hum Commun Res 2003, 29:43I-447.

42. Tutzauer $F$ : On the sensible application of familywise alpha adjustment. Hum Commun Res 2003, 29:455-463.

43. Pitkänen $T$, Lyyra AL, Pulkkinen $L$ : Age of onset of drinking and the use of alcohol in adulthood: a follow-up study from age 842 for females and males. Addiction 2005, 100:652-66I.

44. Bonomo YA, Bowes G, Carolyn C, Carlin JB, Patton GC: Teenage drinking and the onset of alcohol dependence: a cohort study over seven years. Addiction 2004, 99:1520-1528.

45. Finkelstein JW, Von Eye A, Preece MA: The relationship between aggressive behavior and puberty in normal adolescents: a longitudinal study. J Adolesc Health 1994, 15:319-26.

46. Sontag LM, Graber JA, Brooks-Gunn J, Warren MP: Coping with Social Stress: Implications for Psychopathology in Young Adolescent Girls. J Abnorm Child Psychol 2008, 36:I I59-II 74.

47. Butland B, Jebb S, Kopelman P, McPherson K, Thomas S, Mardell J, Parry V: Foresight tackling obesities: future choices project report 2nd edition. London: Department of Innovation Universities and Skill; 2007.
48. Neovius M, Sundström J, Rasmussen F: Combined effects of overweight and smoking in late adolescence on subsequent mortality: nationwide cohort study. BMJ 2009, 338:b496.

49. Ridler C, Townsend N, Dinsdale H, Mulhall C, Rutter H: National child measurement programme: detailed analysis of the 2007/08 national dataset Oxford: National Obesity Observatory; 2009.

50. World Health Organisation: European strategy for child and adolescent health and development. Copenhagen 2005.

\section{Pre-publication history}

The pre-publication history for this paper can be accessed here:

\section{http://www.biomedcentral.com/1471-2458/9/446/pre} pub
Publish with BioMed Central and every scientist can read your work free of charge

"BioMed Central will be the most significant development for disseminating the results of biomedical research in our lifetime. "

Sir Paul Nurse, Cancer Research UK

Your research papers will be:

- available free of charge to the entire biomedical community

- peer reviewed and published immediately upon acceptance

- cited in PubMed and archived on PubMed Central

- yours - you keep the copyright
BioMedcentral 